\section{Secondary Transmission of Coronavirus Disease from Presymptomatic Persons, China}

\author{
Weiwei Zhang, ${ }^{1}$ Weibin Cheng, ${ }^{1}$ Lei Luo, ${ }^{1}$ Yu Ma, \\ Conghui Xu, Pengzhe Qin, Zhoubin Zhang \\ Author affiliations: Guangzhou Center for Disease Control and \\ Prevention, Guangzhou, China (W. Zhang, L. Luo, Y. Ma, C. Xu, \\ P. Qin, Z. Zhang); Guangdong Second Provincial General \\ Hospital, Guangzhou (W. Cheng)
}

DOI: https://doi.org/10.3201/eid2608.201142

We explored the secondary attack rate in different types of contact with persons presymptomatic for coronavirus disease (COVID-19). Close contacts who lived with or had frequent contact with an index case-patient had a higher risk for COVID-19. Our findings provide population-based evidence for transmission from persons with presymptomatic COVID-19 infections.

Coronavirus disease (COVID-19) caused by severe acute respiratory syndrome coronavirus 2 (SARS$\mathrm{CoV}-2)$ is rapidly spreading across the globe. Some case reports and modeling studies suggest asymptomatic carriage of SARS-CoV-2 plays a role in transmission (1-3). Studies have shown that $30 \%-59 \%$ of SARS-CoV-2 infections are asymptomatic $(3,4)$, which poses tremendous infection control challenges. To control asymptomatic infections, China implemented active case surveillance and enhanced social distancing measures, which include contact tracing, quarantine for key populations, medical observation, and curtailed social activities (5). However, additional information on the characteristics of presymptomatic transmission is needed to develop targeted control and prevention guidance.

We analyzed contact-tracing surveillance data collected during January 28-March 15, 2020, to explore the secondary attack rate from different types of contact with persons presymptomatic for COVID-19 in Guangzhou, China. Asymptomatic COVID-19 cases were found mainly through close contact screening, clustered epidemic investigations, follow-up investigation of infection sources, and active surveillance of key populations with travel or residence history in areas with continuous transmission of COVID-19 in China and abroad. We developed a case definition for presymptomatic COVID-19, criteria for close contact, and contact investigation and management

${ }^{1}$ These first authors contributed equally to this article. guidelines (Appendix, https:/ / wwwnc.cdc.gov/EID/ article/26/8/20-1142.pdf). We estimated secondary attack rate (SAR) and 95\% CI based on the proportion of COVID-19 incidence among close contacts. We calculated the mean reproductive number $\left(R_{0}\right)$ from the number of secondary infections observed among close contacts of each index case. The study was approved by the ethics committee of Guangzhou Center for Disease Control and Prevention, which granted a waiver for informed consent. Data collection was conducted under the authority of the China Center for Disease Control and Prevention.

As of March 15, a total of 359 COVID-19 cases were confirmed in Guangzhou. Among them, 83 (23\%) persons were asymptomatic at diagnosis; 71 (86\%) of whom later developed symptoms. Among presymptomatic cases, 38 had $\geq 1$ (range 1-90, median 4) close contact. We identified and included 369 close contacts in this study. Median age of close contacts was 35 years (range 0-93 years), 23.8\% were family members of an index case, and 12 were confirmed to be infected via nucleic acid testing. Among them, 8 close contacts developed symptoms, and 4 were asymptomatic at the time of this study (Appendix Table).

The overall SAR was 3.3\% (95\% CI 1.9\%-5.6\%). The SAR among household contacts was $16.1 \%$ and was $1.1 \%$ for social contacts, and 0 for workplace contacts. Older close contacts had the highest SAR compared with other age groups; $8.0 \%$ in persons $\geq 60$ years of age compared with $1.4 \%-5.6 \%$ in persons $<60$ years of age. Close contacts of asymptomatic index case-patients had the lowest SAR, $0.8 \%$, but the SAR was $3.5 \%$ for those with mild symptoms, $5.7 \%$ for those with moderate symptoms, and $4.5 \%$ for those with severe symptoms. Close contacts that lived with an index case-patient had 12 times the risk for infection and those who had frequent contact with an index case-patient, $>5$ contacts during 2 days before the index case was confirmed, had 29 times the risk for infection (Table).

Our findings substantiate previous reports from China and Germany $(1,2,6)$ and show that SARSCoV-2 can be transmitted during asymptomatic COVID-19 infection period. The probability of infection increased substantially among close contacts who shared living environments or had frequent contact with an index case-patient, which underlines the need for prompt contact-based surveillance and social distancing (7). Our results also showed most secondary infections occurred in confined familial clusters and that persons $\geq 60$ years of age appear to be more vulnerable to being infected. These results are consistent with previous reports on epidemiologic 
Table. Characteristics of and secondary attack rates among 369 close contacts of persons with presymptomatic coronavirus disease 2019, China*

\begin{tabular}{|c|c|c|c|c|}
\hline Variable & No. contacts & No. infected & Attack rate, $\%(95 \% \mathrm{Cl})$ & Relative risk $(95 \% \mathrm{Cl})$ \\
\hline \multicolumn{5}{|c|}{ 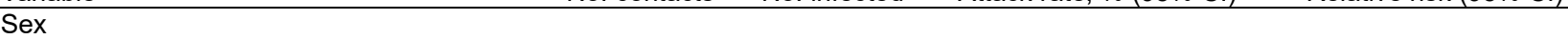 } \\
\hline$M$ & 217 & 5 & $2.3(0.1-5.2)$ & Referent \\
\hline $\mathrm{F}$ & 152 & 7 & $4.6(2.2-8.9)$ & $2.1(0.6-6.6)$ \\
\hline \multicolumn{5}{|l|}{ Age } \\
\hline$\leq 17$ & 46 & 2 & $4.3(1.2-14.5)$ & Referent \\
\hline $18-30$ & 104 & 3 & $2.9(1.0-8.1)$ & $0.7(0.1-4.1)$ \\
\hline $31-40$ & 72 & 1 & $1.4(0.2-7.4)$ & $0.4(0.03-3.5)$ \\
\hline $41-50$ & 68 & 1 & $1.5(0.3-7.9)$ & $0.4(0.03-3.7)$ \\
\hline $51-60$ & 54 & 3 & $5.6(1.9-15.1)$ & $1.3(0.2-8.1)$ \\
\hline$\geq 61$ & 25 & 2 & $8.0(1.4-27.5)$ & $1.9(0.3-14.5)$ \\
\hline \multicolumn{5}{|l|}{ Index case-patient status ${ }^{*}$} \\
\hline Asymptomatic & 119 & 1 & $0.8(0.2-5.6)$ & Referent \\
\hline Mild symptoms & 141 & 5 & $3.5(1.5-8.0)$ & $4.3(0.5-37.7)$ \\
\hline Moderate symptoms & 87 & 5 & $5.7(2.5-12.8)$ & $7.2(0.8-62.7)$ \\
\hline Severe symptoms & 22 & 1 & $4.5(0.8-21.8)$ & $5.6(0.3-93.4)$ \\
\hline \multicolumn{5}{|l|}{ Contact mode } \\
\hline Social interaction with friends or relatives & 66 & 1 & $1.5(0.3-8.1)$ & Referent \\
\hline Lived together & 62 & 10 & $16.1(9.0-27.2)$ & $12.5(1.6-100.8)$ \\
\hline Worked together & 119 & 0 & 0 & 0 \\
\hline Social interaction with strangers & 122 & 1 & $0.8(0.2-4.9)$ & $0.5(0.03-8.7)$ \\
\hline \multicolumn{5}{|l|}{ Contact frequency† } \\
\hline Rare & 149 & 1 & $0.7(0.1-3.7)$ & Referent \\
\hline Moderate & 159 & 1 & $0.6(0.1-3.5)$ & $0.9(0.1-15.1)$ \\
\hline Frequent & 61 & 10 & $16.4(9.2-27.6)$ & $29.0(3.6-232.3)$ \\
\hline \multicolumn{5}{|c|}{$\begin{array}{l}\text { *Status as of March } 30,2020 \text {, based on the person's clinical course assessed by a physician. Moderate symptoms included fever, respiratory symptoms, } \\
\text { and radiographic evidence of pneumonia. Severe symptoms included breathing rate } \geq 30 / \mathrm{min} \text {; oxygen saturation level } \leq 93 \% \text { at rest; oxygen concentration } \\
\text { level } \mathrm{PaO}_{2} / \mathrm{FiO} \mathrm{O}_{2} \leq 300 \mathrm{mmHg}(1 \mathrm{mmHg}=0.133 \mathrm{kPa}) ; \text { lung infiltrates }>50 \% \text { within the past } 24-48 \mathrm{~h} \text {; respiratory failure requiring mechanical ventilation; } \\
\text { septic shock; or multiple organ dysfunction or failure. All other symptomatic cases were classified as mild. } \\
\text { †Rare contact was defined as contacted with index cases }<2 \text { times during } 2 \text { days preceding confirmation of infection. Moderate contact was defined as } \\
\text { contacted with index cases } 3-5 \text { times during } 2 \text { days preceding confirmation of infection. Frequent contact was defined as contacted with index cases } \geq 5 \\
\text { times during } 2 \text { days preceding confirmation of infection. }\end{array}$} \\
\hline
\end{tabular}

characteristics of 72,314 COVID-19 cases in China (8) and suggest that household-based isolation should be cautiously implemented for persons with asymptomatic suspected cases. We also noted that persons with asymptomatic infections appeared to be less effective in transmitting the virus. However, this finding should not discourage isolation and surveillance efforts. The $\mathrm{R}_{0}$ in this cohort was 0.3 (95\% CI 0.2-0.5), which was far smaller than the overall $\mathrm{R}_{0}$ of 2.2 reported previously (9). This low transmission level could be the result of active surveillance, centralized quarantine, and forceful social-distancing strategies in Guangzhou.

Interpretation of the findings should be taken with caution, and several limitations influence our estimation of the SAR. First, the number of close contacts was limited because we only included those who had been reached, and asymptomatic infections might have been missed. Second, we excluded close contacts who were exposed to $\geq 2$ confirmed COVID-19 case-patients. Third, the presymptomatic transmission period is not well defined.

Despite these limitations, our analysis provides valuable information on secondary transmission of SARS-CoV-2 in different types of contact with presymptomatic COVID-19 case-patients. Further evidence is needed to define the population characteristics, communicable period, and the volume and duration of viral shedding from persons with asymptomatic infections.

\section{Acknowledgments}

We thank Weiming Tang for reviewing and editing the article.

This study was supported by funding from the Project for Key Medicine Discipline Construction of Guangzhou Municipality (grant no. 2017-2019-07).

\section{About the Author}

Dr. W. Zhang is a senior public health physician at the Guangzhou Center for Disease Control and Prevention. Her research interests are health promotion and infectious disease epidemic modeling.

\section{References}

1. Bai Y, Yao L, Wei T, Tian F, Jin DY, Chen L, et al. Presumed asymptomatic carrier transmission of COVID-19. JAMA. 2020 Feb 21 [Epub ahead of print]. https:/ / doi.org/10.1001/ jama.2020.2565

2. Rothe C, Schunk M, Sothmann P, Bretzel G, Froeschl G, Wallrauch C, et al. Transmission of 2019-nCoV Infection 
from an Asymptomatic Contact in Germany. N Engl J Med. 2020;382:970-1. https:// doi.org/10.1056/NEJMc2001468

3. Nishiura H, Linton NM, Akhmetzhanov AR. Serial interval of novel coronavirus (COVID-19) infections. Int J Infect Dis. 2020;93:284-6. https://doi.org/10.1016/j.ijid.2020.02.060

4. Pan A, Liu L, Wang C, Guo H, Hao X, Wang Q, et al. Association of public health interventions with the epidemiology of the COVID-19 outbreak in Wuhan, China. JAMA. 2020 Apr 10 [Epub ahead of print]. https://doi.org/ 10.1001/jama.2020.6130

5. National Health Commission. Questions and answers about the prevention and control of asymptomatic cases infected by 2019-nCoV [in Chinese] 2020. [cited 2020 April 24]. http:/ / www.nhc.gov.cn/jkj/s3578/202003/718c79c96f3e464 09dd49303d41a00ef.shtml

6. Tong ZD, Tang A, Li KF, Li P, Wang HL, Yi JP, et al. Potential presymptomatic transmission of SARS-CoV-2, Zhejiang Province, China, 2020. Emerg Infect Dis. 2020;26:1052-4. https://doi.org/10.3201/eid2605.200198

7. Wang FS, Zhang C. What to do next to control the $2019-\mathrm{nCoV}$ epidemic? Lancet. 2020;395:391-3. https:/ / doi.org/10.1016/ S0140-6736(20)30300-7

8. Novel Coronavirus Pneumonia Emergency Response Epidemiology Team. Vital surveillances: the epidemiological characteristics of an outbreak of 2019 novel coronavirus diseases (COVID-19) - China, 2020. China CDC Weekly. 2020;2: 113-122 [cited 2020 April 24]. http:/ / weekly.chinacdc. cn/en/article/id/e53946e2-c6c4-41e9-9a9b-fea8db1a8f51

9. Li Q, Guan X, Wu P, Wang X, Zhou L, Tong Y, et al. Early transmission dynamics in Wuhan, China, of novel coronavirus-infected pneumonia. N Engl J Med. 2020;382:1199-207. https://doi.org/10.1056/NEJMoa2001316

Addresses for correspondence: Zhoubin Zhang or Pengzhe Qin, Guangzhou Center for Disease Control and Prevention, No.1 Qide road, Guangzhou, 510440, China; email gzcdczzb@gzcdc.org.cn or petgyy@gmail.com

\section{Abdominal Visceral Infarction in 3 Patients with COVID-19}

Giulia Besutti, Riccardo Bonacini, Valentina lotti, Giulia Marini, Nicoletta Riva, Giovanni Dolci, Mariarosa Maiorana, Lucia Spaggiari, Filippo Monelli, Guido Ligabue, Giovanni Guaraldi, Paolo Giorgi Rossi, Pierpaolo Pattacini, Marco Massari

Author affiliations: Azienda Unità Sanitaria Locale di Reggio Emilia-Istituto di Ricovero e Cura a Carattere Scientifico di Reggio Emilia (AUSL-IRCCS), Reggio Emilia, Italy (G. Besutti,
R. Bonacini, V. lotti, G. Marini, N. Riva, G. Dolci, M. Maiorana, L. Spaggiari, P.G. Rossi, P. Pattacini, M. Massari); University of Modena and Reggio Emilia Clinical and Experimental Medicine PhD Program, Modena, Italy (G. Besutti, M. Maiorana); University of Modena and Reggio Emilia, Modena (R. Bonacini, G. Dolci, F. Monelli, G. Ligabue, G. Guaraldi)

DOI: https://doi.org/10.3201/eid2608.201161

A high incidence of thrombotic events has been reported in patients with coronavirus disease (COVID-19), which is caused by severe acute respiratory syndrome coronavirus-2 (SARS-CoV-2) infection. We report 3 clinical cases of patients in Italy with COVID-19 who developed abdominal viscera infarction, demonstrated by computed tomography.

$\mathrm{F}_{\mathrm{e}}^{\mathrm{r}}$ requent thrombotic events, mostly pulmonary embolisms, have been reported in patients with coronavirus disease (COVID-19) (1-4). We describe 3 cases of COVID-19 complicated by abdominal visceral infarction that occurred in inhabitants of the Emilia Romagna region in northern Italy.

Patient 1, a 54-year-old male former smoker with a history of asthma and quiescent ulcerative colitis not receiving any treatment, was admitted to the emergency department (ED) on February 28, 2020, for syncope. He was discharged after undergoing chest radiography and brain computed tomography $(\mathrm{CT})$, the results of which were unremarkable. He returned to the ED after 5 days for treatment of dyspnea, fatigue, and fever. Blood tests revealed decreased oxygen saturation $(94 \%)$, increased C-reactive protein $(\mathrm{CRP})$ level $(5.38 \mathrm{mg} / \mathrm{dL}$; reference $<0.5$ $\mathrm{mg} / \mathrm{dL})$, and lymphopenia $\left(0.69 \times 10^{3}\right.$ cells $/ \mathrm{mm}^{3}$; reference range $0.8-4 \times 10^{3}$ cells $\left./ \mathrm{mm}^{3}\right)$. Chest CT scan demonstrated bilateral viral pneumonia, and nasopharyngeal and oropharyngeal swab specimens were positive for severe acute respiratory syndrome coronavirus 2 (SARS-CoV-2). He was hospitalized and treated with lopinavir/ritonavir $(400 / 100 \mathrm{mg}$ orally $2 \times / \mathrm{d}$ ), and hydroxychloroquine (200 mg orally $2 \times / d)$. He was discharged to home after 3 hospital days, on therapy; no anticoagulant prophylaxis was suggested. He was rehospitalized 6 days after discharge when he developed sharp right flank and lumbar pain, fever, and dysuria. Blood and urine tests revealed neutrophilia $\left(9.9 \times 10^{3}\right.$ cells $/ \mathrm{mm}^{3}$; reference range $1.6-7.5 \times 10^{3}$ cells $\left./ \mathrm{mm}^{3}\right)$, increased lactate dehydrogenase $(\mathrm{LDH})(1,507 \mathrm{U} / \mathrm{L}$; reference range $28-378 \mathrm{U} / \mathrm{L})$, increased CRP $(1.43 \mathrm{mg} / \mathrm{dL})$, and proteinuria $(50 \mathrm{mg} / \mathrm{dL})$. CT scan demonstrated a large right kidney arterial infarction (Figure, panel A). He was treated with low molecular weight 